\title{
Adult Acute Myeloid Leukemia without Maturation
}

National Cancer Institute

\section{Source}

National Cancer Institute. Adult Acute Myeloid Leukemia without Maturation. NCI

Thesaurus. Code $C 9380$.

An acute myeloid leukemia without maturation occurring in adults. 